\title{
AVALIAÇÃO DA FREQUÊNCIA DE Balantidium coli EM SUÍNOS, TRATADORES DE SUÍNOS E PRIMATAS NÃO HUMANOS NO ESTADO DO RIO DE JANEIRO
}

\author{
Alynne da Silva Barbosal, Otilio Machado Pereira Bastos ${ }^{2}$, Claudia M. \\ Antunes Uchôa ${ }^{2}$, Laís Verdan Dib ${ }^{1}$ e Maria Regina Reis Amendoeira ${ }^{1}$
}

\section{RESUMO}

Este trabalho relata a frequência de Balantidium coli nos tratadores de suínos e primatas não humanos que trabalham em criatórios localizados em distintos municípios do estado do Rio de Janeiro. No período de 2012 a 2014, foram coletadas 104 amostras fecais de 52 tratadores, 790 amostras fecais de suínos e 1.115 de primatas não humanos. Todas as amostras foram coletadas sem conservante químico, sendo submetidas às técnicas de Faust et al., Sheather modificada, Ritchie modificada, Lutz e exame direto. Formas evolutivas de B. coli não foram detectadas nas amostras fecais dos tratadores, porém foram evidenciadas em 49,2\% dos suínos e em 41,5\% do material fecal dos símios. A ausência da balantidiose em humanos, embora estivessem em contato próximo com animais parasitados, como foi demonstrado neste estudo, pode ter ocorrido em virtude da adoção de adequadas medidas higiênico-sanitárias na manipulação desses animais ou graças à resistência humana à infecção por esse protozoário.

DESCRITORES: Balantidiose; tratadores de animais; zoonose; parasitoses intestinais.

\section{ABSTRACT}

Balantidium coli: frequency evaluation in pig, pig handlers and nonhuman primate in the state of Rio de Janeiro, Brazil.

This paper reports the frequency of Balantidium coli in pig, pig handlers and nonhuman primate working on farms located in different municipalities in the state of Rio de Janeiro. From 2012 to 2014,104 fecal samples were collected from 52 handlers, 790 fecal samples from pigs and 1,115 from nonhuman primates. All samples were collected free of chemical preservatives and then underwent the following techniques: Faust et al., Sheather modified, Ritchie modified and Lutz, as well as direct examination. Evolutionary forms of B. coli were not detected in the fecal samples from the handlers, but were found in $49.2 \%$ of the fecal material from pigs, and in $41.5 \%$. from apes. The absence of balantidiosis in humans, in spite of being in close contact

\footnotetext{
1. Laboratório de Toxoplasmose, Instituto Oswaldo Cruz, Fundação Oswaldo Cruz, Rio de Janeiro, Brasil.

2. Departamento de Microbiologia e Parasitologia, Instituto Biomédico, Universidade Federal Fluminense, Niterói, Rio de Janeiro, Brasil.
}

Autor correspondente: Alynne da Silva Barbosa, Laboratório de Toxoplasmose, IOC, Avenida Brasil, 4365, Manguinhos, CEP 21045-900, Rio de Janeiro, Brasil. E-mail: alynnedsb@gmail.com 
with infected animals, as demonstrated in this study, may be due to the adoption of adequate sanitary measures for handling the animals or even due to human resistance to infection by this parasite.

KEY WORDS: Balantidiosis; animal handlers; zoonosis; intestinal parasites.

\section{INTRODUÇÃO}

Balantidium coli é o único protozoário ciliado que infecta os humanos, embora possa parasitar uma grande diversidade de animais. Suínos e primatas não humanos são considerados os reservatórios mais importantes da parasitose, favorecendo a transmissão zoonótica (Nakauchi, 1999; Walzer \& Healy, 1982). A infecção é adquirida de forma indireta pela ingestão de cistos que tenham contaminado água e alimentos ou pelo contato direto com hospedeiros infectados, o que torna médicos veterinários e tratadores de animais os principais grupos de risco (Schuster \& Ramirez-Avila, 2008; Woody \& Woody, 1960).

O protozoário é essencialmente um parasito de intestino grosso, que habita principalmente as regiões de ceco e cólon (Schuster \& Ramirez-Avila, 2008; Zaman, 1978). O parasito pode causar, nos hospedeiros infectados, manifestações clínicas que variam desde casos assintomáticos até quadros clínicos de gastroenterites severas com episódios de disenteria, que podem evoluir para o óbito (Dorfman et al., 1984; Schuster \& Ramirez-Avila, 2008; Vásquez \& Vidal, 1999).

Infecções por $B$. coli foram relatadas em diversas partes do mundo; os maiores focos endêmicos foram documentados nas Américas Central e do Sul; Filipinas; Papua Nova Guiné; região da antiga Pérsia; Ásia Central e do Sul e algumas ilhas do Pacífico (Solaymani-Mohammadi \& Petri Jr, 2006; Zaman, 1978).

As informações sobre a ocorrência da balantidiose humana são escassas (Hernández \& Rivera, 1991). No Brasil, Machado et al. (1969) relataram a frequência da parasitose em $0,7 \%$ dos pacientes atendidos no município de Niterói, Rio de Janeiro. Dos nove pacientes diagnosticados, somente dois tinham contato com suínos. Em hospitais no estado do Pará, Vasconcelos (1981) relatou $0,9 \%$ de positividade para B. coli em 110 crianças que apresentavam quadro clínico de diarreia aguda. Em 2005, em estudo de levantamento de dados secundários de prontuários de pessoas atendidas em hospitais no município de Concórdia, Santa Catarina, Marques et al. (2005) demonstraram a presença do parasito em $0,2 \%$ dos casos atendidos. Já Pereira (2010), estudando as parasitoses em escolares em Jequié na Bahia, evidenciou $0,7 \%$ de positividade para o protozoário. 
B. coli é considerado um patógeno negligenciado, uma vez que é pouco estudado (Walzer et al., 1982). Walzer et al. (1973) ressaltaram que a maioria dos relatos de balantidiose humana são de casos clínicos de indivíduos sintomáticos, casos com complicações ou fatais, sendo poucos os relatos de surtos ou inquéritos coproparasitológicos específicos para pesquisa deste protozoário. É notória a escassez de informações sobre a epidemiologia deste parasito e indiscutível a constatação de que os tratadores de animais constituem grupo de risco de aquisição da balantidiose, em razão da proximidade com suínos e símios, considerados reservatórios desse protozoário. Diante disso, este estudo teve como objetivo determinar a frequência de $B$. coli em material fecal de tratadores de suínos e primatas não humanos que trabalham em criatórios localizados em diferentes municípios do estado do Rio de Janeiro.

\section{MATERIAL E MÉTODOS}

O estudo foi conduzido em nove granjas de suínos e em dois criatórios de primatas não humanos localizados em diferentes municípios do estado do Rio de Janeiro. Foram convidadas a participar do estudo granjas de suínos do tipo familiar, localizadas no interior da região metropolitana e baixada litorânea, visto que a maioria dos produtores familiares (tradicionais) se concentra nestas regiões. As granjas industriais selecionadas são as principais produtoras de suínos no estado do Rio Janeiro, especialmente a do município de Petrópolis. As granjas que aceitaram participar do estudo estão situadas nas cidades de Itaboraí, Maricá, Petrópolis, Rio Bonito, Saquarema, Silva Jardim, Casimiro de Abreu e Itaperuna. Os criatórios dos primatas não humanos incluídos na pesquisa foram o Setor de Criação de Animais de Laboratório (Cecal), situado no bairro de Manguinhos, dentro da Fundação Oswaldo Cruz, e o Centro de Primatologia do Estado do Rio de Janeiro (CPRJ/INEA), localizado no município de Guapimirim.

A pesquisa de $B$. coli foi realizada no período de agosto de 2012 a janeiro de 2014. O inquérito parasitológico envolveu 104 amostras fecais dos tratadores de suínos e primatas não humanos, 790 amostras de suínos e 1.115 dos símios cativos. O estudo obteve parecer favorável do Comitê de Ética Humano (CEP/IOC) em 18 de junho de 2012, Protocolo $n^{\circ}$ 648/12, e do Comitê de Ética Animal (CEUA-Fiocruz) em 02 de julho de 2012, Licença ${ }^{\circ}$ LW57/12, Protocolo n ${ }^{\circ}$ P79/11-2, e do SISBIO - IBAMA, Protocolo n ${ }^{\circ} 31900-$ 2. Após esclarecimentos sobre o estudo, os tratadores que aceitaram participar assinaram o termo de consentimento livre e esclarecido (TCLE).

Os participantes receberam um kit de coleta de fezes que continha dois coletores fecais de cor branca com capacidade de $80 \mathrm{~mL}$, etiquetados e sem conservante, duas pazinhas de madeira primoutilizadas e um informe detalhando o procedimento de coleta. Explicou-se, verbalmente, a cada 
pessoa que a amostra deveria ser coletada em dois dias distintos, armazenadas em temperatura de geladeira e identificadas com nome e data de coleta. $\mathrm{O}$ recolhimento dos coletores foi realizado de forma programada com cada tratador.

Nas propriedades familiares de suínos, os tratadores eram, predominantemente, os proprietários dos animais e, em alguns casos, membros de sua família. Na granja familiar de Rio Bonito, aderiram ao estudo cinco pessoas (100\%); em Araruama, uma (100\%); em Saquarema, três (100\%); em Maricá, duas (100\%); em Itaboraí, uma (50\%); em Silva Jardim, uma (100\%) e em Casimiro de Abreu, duas (100\%). Nas granjas industriais, o grupo dos tratadores foi composto por auxiliares de manejo e, em alguns casos, como em Itaperuna, também pelos proprietários. Na granja de Petrópolis, seis $(60 \%)$ pessoas participaram e, em Itaperuna, quatro (100\%). Nos criatórios de símios, o grupo de tratadores era composto, predominantemente, por auxiliares de manejo e, em alguns casos, por médicos veterinários. Nos CPRJ/INEA, 14 $(77,7 \%)$ pessoas participaram do estudo e, no Cecal/Fiocruz, foram $13(50 \%)$ os participantes.

Também foram coletadas 790 amostras fecais de suínos, sendo: 88 de propriedades familiares dos municípios de Rio Bonito (21), Araruama (7), Saquarema (12), Maricá (8), Itaboraí (12), Casimiro de Abreu (23) e Silva Jardim (5) e 702 de granjas industriais de Petrópolis (479) e Itaperuna (223). No estudo, foi incluída uma granja de cada município, totalizando sete granjas familiares e duas do tipo industrial. As amostras fecais dos suínos foram coletadas de forma pontual em cada granja, individualmente, diretamente da ampola retal com luva de palpação longa, sendo posteriormente identificadas e acondicionadas em recipiente isotérmico.

Para fins de amostragem, nas granjas familiares, optou-se por fazer a coleta do máximo de animais, pois o número de suínos em cada propriedade era pequeno, resultando em diferença no número total de amostras de cada granja. Foram excluídas as fêmeas prenhes, recém-paridas e leitões recém-nascidos. Nas granjas do tipo industrial, foi utilizado o intervalo de confiança de, no mínimo, $80 \%$ com $5 \%$ de precisão. Neste caso, a exceção foram os setores de terminação e creche da granja de Petrópolis, onde foi adotada amostragem por conveniência, sendo coletadas, no mínimo, 100 amostras fecais de cada categoria, visto que ali havia mais de 1.000 animais em cada setor. Nas propriedades familiares estudadas, os suínos não possuíam raça definida e permaneciam em baias coletivas. Já nas granjas do tipo industrial, os suínos eram oriundos de linhagens específicas. Em Petrópolis, os animais pertenciam à linhagem TOPIG do Brasil ${ }^{\mathbb{R}}$ e, em Itaperuna, à linhagem Agroceres ${ }^{\circledR}$. Nessas, os suínos eram categorizados de acordo com a idade e a função reprodutiva, sendo mantidos em setores específicos. Nesses setores havia baias onde os animais eram divididos de forma homogênea, por lotes. 
Quanto aos primatas não humanos cativos, optou-se por coletar as amostras fecais do assoalho de seus recintos, minimizando o estresse consequente do processo de contenção animal. Ao todo foram coletadas 1.115 amostras fecais dos símios, sendo 960 do Cecal e 155 do CPRJ. Foram representadas, neste estudo, 36 espécies de primatas do Novo Mundo (NM) e duas do Velho Mundo (VM). Em ambas as instituições, os símios ficam abrigados em recintos coletivos, divididos por espécies; todavia, no CPRJ, alguns animais ficam em recintos individuais.

As coletas foram padronizadas de acordo com cada local estudado. Nos recintos do Cecal, há um grande número de animais com pouca variedade de espécies. Neste setor, as coletas foram feitas de forma pontual. A quantidade de amostras coletadas foi idêntica à quantidade de primatas que habitavam cada recinto. No CPRJ, era grande a variedade de espécies de primatas. Neste local, a maioria dos símios pertence aos gêneros Mico, Saguinus, Callithrix e Leontopithecus, sendo, portanto, primatas de menor porte que defecam um pequeno volume fecal. Para aumentar o volume da amostra e com isso garantir a realização com qualidade das técnicas laboratoriais, optou-se por coletar o material de cada recinto em triplicata (três dias consecutivos). As triplicatas foram consideradas uma única amostra.

Para as coletas deu-se preferência a conteúdos fecais frescos, que foram coletados pelos próprios tratadores no período da manhã, momento em que os recintos são rotineiramente higienizados. As amostras foram depositadas em coletores plásticos de $80 \mathrm{~mL}$ sem conservante químico, sendo posteriormente acondicionados em recipientes isotérmicos.

Todas as amostras fecais foram encaminhadas ao Laboratório de Parasitologia do Instituto Biomédico da Universidade Federal Fluminense para análise. Uma parte do material fecal foi imediatamente processada pelo exame direto para identificar, principalmente, a presença de trofozoítos de $B$. coli e também de cistos (Figura). No momento do exame, o bolo fecal foi submetido à retirada de alíquotas de vários pontos, tanto da superfície como em profundidade. A raspagem do bolo fecal foi sempre realizada com o auxílio de um bastão de vidro. O material fecal oriundo desta raspagem foi homogeneizado em solução salina tamponada e estéril. Uma alíquota desta solução fecal foi transferida para lâmina de microscopia coberta com lamínula 24 X $32 \mathrm{~mm}$, sendo, então, encaminhada para leitura em microscópio óptico.

A outra parte da amostra foi homogeneizada e o filtrado, aliquotado em tubos de centrífuga de $15 \mathrm{~mL}$, de fundo cônico, para a realização das seguintes técnicas: centrífugo-sedimentação de Ritchie (1948), modificada por Young et al. (1979) ; centrífugo-flutuação de Faust et al. (1938) e centrífugo-flutuação de Sheather (1923), modificada por Huber et al. (2003). Parte do filtrado foi submetida à técnica de sedimentação espontânea de Lutz (1919). Foi sempre produzida uma lâmina de microscopia por cada técnica realizada. A leitura dessas lâminas foi realizada em microscópio óptico Olympus ${ }^{\circledR}$ BX 41, inicialmente com objetiva de 10X e, para confirmação, se necessária, foi utilizada objetiva de 40X. 

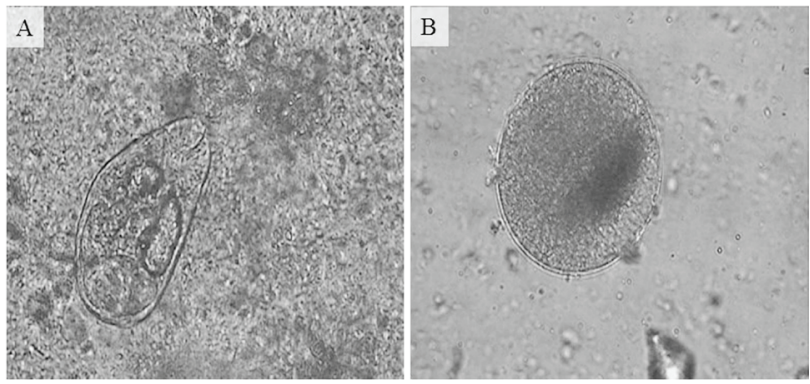

Figura. Formas evolutivas de Balantidium coli detectadas nas amostras fecais dos suínos e primatas não humanos. Trofozoíto de $B$. coli em objetiva de $40 \mathrm{X}$ detectado em amostra fecal de primata não humano. Cisto de $B$. coli em objetiva de 40X diagnosticado em amostra fecal de suíno.

\section{RESULTADOS}

Foram processadas 104 amostras fecais humanas, correspondentes a 52 tratadores de animais. Formas evolutivas de B. coli não foram diagnosticadas nas amostras dos tratadores de suínos e de primatas não humanos, porém foram detectadas em $49,4 \%$ e $41,5 \%$ das amostras fecais coletadas de suínos e primatas não humanos, respectivamente (Tabela).

Tabela. Frequência de Balantidium coli em amostras fecais de suínos, primatas não humanos e seus tratadores

\begin{tabular}{ccc}
\hline & Total de amostras & Balantidium coli \\
\hline Tratadores & 104 & 0 \\
Suínos & 790 & $389(49,2 \%)$ \\
Primatas não humanos & 1.115 & $463(41,5 \%)$ \\
\hline
\end{tabular}

Embora não fosse este o objetivo do estudo, foram diagnosticados cinco tratadores com amostras fecais positivas para outras espécies de protozoários e dois com formas evolutivas de helmintos, totalizando nove amostras positivas para protozoários e quatro para helmintos. Estas formas evolutivas foram diagnosticadas em amostras fecais de três tratadores do Centro de Primatologia de Guapimirim e nas propriedades familiares de suíno de Rio Bonito, Silva Jardim e Casimiro de Abreu. A frequência dos parasitos foi de 3,8\% de ancilostomídeos, $1,9 \%$ de Trichuris trichiura e 3,8\% de cistos do complexo Entamoeba histolytica/Entamoeba dispar em amostras do Centro 
de Primatologia de Guapimirim. A associação entre Entamoeba coli e Giardia duodenalis apresentou frequência de 1,9\%, sendo diagnosticada em amostra de Rio Bonito; obteve-se 3,8\% de positividade para Endolimax nana, detectada em Silva Jardim e Casimiro de Abreu.

\section{DISCUSSÃO}

Todas as amostras fecais analisadas dos tratadores dos animais, tanto dos que tinham contato com suínos quanto dos que tratavam de primatas não humanos, resultaram negativas para formas evolutivas de B. coli, embora tenha sido detectada elevada frequência do parasito no material fecal dos animais. Elevado índice (acima de 60\%) de B. coli em amostras de suínos e resultado negativo nos exames de fezes dos seus criadores também foram relatados em comunidades da Venezuela por Guzmán et al. (2013).

A infecção por $B$. coli tem distribuição em nível mundial, mas sua incidência em humanos é pouco frequente. Até 1980, foram descritos na literatura 1.000 casos humanos (Solaymani-Mohammadi \& Petri Jr, 2006). Apesar da baixa frequência, estudos coproparasitológicos realizados na América Latina têm demonstrado a positividade da balantidiose em humanos, sobretudo entre os que entram em contato com suínos. Esteban et al. (1998) demonstraram positividade entre $1,2 \%$ e $5,3 \%$ para $B$. coli em escolares de comunidades rurais na Bolívia e $54 \%$ de positividade nas amostras de suínos dessas comunidades. Mesmo não tendo avaliado as amostras dos animais, Castillo (2013) evidenciou o parasito em 5\% de 100 produtores de suínos na província de Balsas, localizada na região sul do Equador. Todos esses resultados diferem do detectado no presente estudo.

Segundo Hernández \& Rivera (1991), a literatura sobre esta parasitose é escassa por duas razões fundamentais: a primeira diz respeito à baixa prevalência do parasito em humanos; a segunda, ao número reduzido de publicações, que comumente estão voltadas para casos clínicos isolados. Geralmente, os editores de muitas revistas se mostram receosos quanto à aceitação de artigos sobre casos clínicos, sendo, portanto, publicados como notas curtas, frequentemente em espanhol nos países latinos, em revistas de pouca circulação. Com isso, essa literatura torna-se pouco conhecida em nível mundial. As revisões de literatura, trazem mais casos de países europeus ou norte-americanos, onde geralmente a parasitose tem baixos índices de prevalência. Este fato pode gerar uma falsa informação de que a balantidiose seja uma parasitose rara.

A balantidiose pode ser considerada uma doença de transmissão zoonótica, já que em muitos casos clínicos relatados em humanos foi relatada a existência do contato humano-animais (Solaymani-Mohammadi \& Petri Jr, 2006). Este fato não foi evidenciado no presente estudo, embora haja sido 
elevada a frequência do protozoário em amostras fecais de suínos e primatas não humanos. Entretanto, já foram descritos casos de transmissão nos quais não houve participação de animais como fonte da infecção, como os relatados em populações muçulmanas (Geddes, 1952), em instituições fechadas, como hospitais psiquiátricos (Elliot e Hotson, 1953), e em pessoas que vivem em ambiente urbano sem contato com animais (Figueiredo et al., 2012).

Geralmente a infecção pelo protozoário em humanos está associada às seguintes circuntâncias: precárias condições sanitárias, dieta pobre em proteínas e rica em carboidratos, acloridria, ingestão elevada de álcool, infecção por bactérias ou outros protozoários, estado de imunocomprometimento e fatores ambientais como umidade e temperatura, que favorecem a viabilidade dos cistos durante várias semanas (Schuster \& Ramirez-Avila, 2008).

Walzer et al. (1973) ressaltaram também que a baixa prevalência do protozoário em humanos pode ser explicada por diferenças antigênicas, ou seja, o homem seria resistente à infecção mesmo vivendo em contato próximo com os animais, em condições socioeconômicas deficientes. A presença marcante das formas evolutivas de B. coli nas amostras fecais dos animais estudados e sua ausência nos exames de seus tratadores, neste caso, pode também estar diretamente relacionada aos hábitos higiênico-sanitários adotados por esses indivíduos, os quais reduzem sua exposição à forma infectante do parasito.

\section{AGRADECIMENTOS}

Ao Centro de Primatologia do Rio de Janeiro (CPRJ/INEA) e Cecal/Fiocruz, por disponibilizar material científico. A Fundação de Amparo à Pesquisa do Estado do Rio de Janeiro (FAPERJ) auxílio número E26/110 440/2012, ao Instituto Oswaldo Cruz (IOC), a Pró-reitoria de Pesquisa e Inovação da Universidade Federal Fluminense (PROPPI-UFF) e a Pró-reitoria de Assuntos Estudantis da Universidade Federal Fluminense (PROAES-UFF).

\section{REFERÊNCIAS}

1. Castillo KES. Identificación de Balantidium coli en personas dedicadas a la porcicultura en el cantón Balsas durante septiembre 2012 a febrero 2013. Ecuador. [Monografia de Carrera de Laboratorio Clínico]. Universidad Nacional de Loja, 2013.

2. Dorfman S, Rangel O, Bravo LG. Balantidiaisis: report of a fatal case with appendicular and pulmonary involvement. Trans R Soc Trop Med Hyg 78: 833-834, 1984.

3. Elliot GB, Hotson RT. Balantidial dysentery. Canad MAJ 69: 317-318, 1953.

4. Esteban JG, Aguirre C, Angles R, Ash LR, Mas-Coma S. Balantidiasis in Aymara children from northern Bolivian altiplano. Am J Trop Med Hyg 59: 922-927, 1998.

5. Faust EC, D'antoni JS, Odon V, Miller MJ, Perez C,Sawitz W, Thomen, LF, Tobie JE, Walker $\mathrm{JH}$ : A critical study of clinical laboratory technics for the diagnosis of protozoan cysts and helminth eggs in feces. I. Preliminary communication. Am J Trop Med 18: 169-183, 1938. 
6. Figueiredo SM, Filippis T, Santos UMP, Caixeta SS, Rocha JFS, Guimarães MM. Report on a Balantidiasis case in a person living with HIV/AIDS(PLWHA). Rev Patol Trop 41:505-509, 2012.

7. Geddes McCA. Balantidiasis in South Persia. Br Med J 1: 629-631, 1952.

8. Guzmán CR, Nessi AP, Gonzáles OH, Hernández MO, Galindo M. Balantidium spp en cerdos y sus criadores: Prevalencia em comunidades de dos Estados de Venezuela. VITAE 54: 1-10, 2013.

9. Hernández F, Rivera P. Balantidiasis: Recopilacion de Conceptos. Rev Costarric Cienc Med 12: 67-75, 1991.

10. Huber F, Bomfim TC, Gomes RS. Comparação da eficiência da técnica de sedimentação pelo formaldeído-éter e da técnica de centrífugo-flutuação modificada na detecção de cistos de Giardia sp. e oocistos de Cryptosporidium sp. em amostras fecais de bezerros. Rev Bras Parasitol Vet 12: 135-137, 2003.

11. Lutz AO. Schistosomum mansoni and Schistomatose according to observations made in Brazil. Mem Inst Oswaldo Cruz 11: 121-155, 1919.

12. Machado O, Pinho AL, Silva S. Aspectos parasitológicos na balantidiose humana. O Hospital 75: 1969-1976, 1969.

13. Marques SMT, Bandeira C, Quadros RM. Prevalência de enteroparasitoses em Concórdia, Santa Catarina, Brasil. Parasitol Latinoam 60: 78-81, 2005.

14. Nakauchi K. The prevalence of Balantidium coli infection in fifty-six mammalian species. $J$ Vet Med Sci 61: 63-65, 1999.

15. Pereira C. Ocorrência da esquistossomose e outras parasitoses intestinais em crianças e adolescentes de uma escola municipal de Jequié, Bahia, Brasil. Rev Saúde Com 1: 24-30, 2010.

16. Ritchie LS. An ether sedimentation technique for routine stool examinations. Bull U S Arm Med Dep 8: 326, 1948.

17. Sheather LA. The detection of intestinal protozoa and mange parasites by a flotation technique. J Comp Pathol Ther 36: 266-275, 1923.

18. Schuster F L, Ramirez-Avila L. Current world status of Balantidium coli. Clin Microbiol Rev 21: 626-638, 2008.

19. Solaymani-Mohammadi S, Petri Jr. WA. Zoonotic implications of the swine Transmittedprotozoal infections. Vet Parasitol 140:189-203, 2006;

20. Vasconcelos JC. Bactérias enteropaotgênicas de diarreia infantil aguda em Tucuruí, Pará. Acta Amazon 11: 527-535, 1981.

21. Vásquez W, Vidal J. Colitis balantidiasica: a proposito de un caso fatal en el departamento de Huancavelica. An Fac Med 60:119-123, 1999.

22. Walzer PD, Judson FN, Murphy KB, Healy GR, English DK, Schultz MG. Balantidiasis outbreak in Truk. Am J Trop Med Hyg 22: 33-41, 1973.

23. Walzer PD, Healy GR. Balantidiasis. In: Steele, JH (Ed), Handbook Series in Zoonoses, CRC Press, Inc., Boca Raton, FL, 1982.

24. Woody NC, Woody HB. Balantidiasis in infancy: Review of the literature and reporto fa case. J Pediatrics 56: 485-489, 1960.

25. Young KH, Bullock SL, Melvin DM, Spruill CL. Ethyl acetate as a substitute for diethyl ether in the formalin-ether sedimentation technique. J Clin Microbiol 10: 852-853, 1979.

26. Zaman V. Balantidium coli. In: Kreier JP (Ed). Parasitic Protozoa. Academic Press, New York, 1978. 